\title{
MicroRNA-30a-5p suppresses tumor cell proliferation of human renal cancer via the MTDH/PTEN/AKT pathway
}

\author{
JIANMIN LI ${ }^{1}$, CHANGYING LI ${ }^{1}$, HONGJIE LI ${ }^{2}$, TING ZHANG ${ }^{1}$, \\ XIAODONG HAO ${ }^{1,3}$, JIWU CHANG ${ }^{1}$ and YONG XU ${ }^{1,3}$
}

\begin{abstract}
${ }^{1}$ Cancer Immunity Research Laboratory, Tianjin Institute of Urology, The Second Hospital of Tianjin Medical University, Tianjin Medical University, Tianjin 300211; ${ }^{2}$ School of Basic Medical Sciences, North China University of Science and Technology, Tangshan, Hebei 063000; ${ }^{3}$ Department of Urology, The Second Hospital of Tianjin Medical University, Tianjin Medical University, Tianjin 300211, P.R. China
\end{abstract}

Received November 23, 2016; Accepted October 2, 2017

DOI: $10.3892 /$ ijmm.2017.3269

\begin{abstract}
The present study aimed to explore the effects of microRNA (miRNA)-30a-5p on tumor proliferation and to seek a potential therapeutic target for the treatment of human renal cancer. The results demonstrated that the expression levels of miRNA-30a-5p were reduced in tumor samples from patients with renal cancer compared with in normal tissue samples. Overall survival and disease-free survival were increased in patients with renal cancer and high miRNA-30a-5p expression compared with in those with low miRNA-30a-5p. Furthermore, overexpression of miRNA-30a-5p suppressed cell proliferation, induced apoptosis, and promoted caspase- $3 / 9$ activities and B-cell lymphoma 2-associated X protein (Bax) protein expression in Caki-2 cells. In addition, the results confirmed that overexpression of miRNA-30a-5p inhibited metadherin (MTDH), upregulated phosphatase and tensin homolog (PTEN) and suppressed phosphorylated (p)-protein kinase B (AKT) protein expression levels in Caki-2 cells. Furthermore, transfection with small interfering RNA-MTDH, increased the effects of miRNA-30a-5p on the inhibition of cell proliferation, and promotion of apoptosis, caspase-3/9 activities and Bax protein expression levels in Caki-2 cells. Knockdown of MTDH expression also upregulated PTEN and suppressed p-AKT protein expression in Caki-2 cells. In conclusion, the present study is the first, to the best of our knowledge, to provide evidence suggesting that miRNA-30a-5p suppresses tumor human renal cancer cell proliferation via the MTDH/PTEN/AKT pathway.
\end{abstract}

Correspondence to: Dr Yong Xu, Department of Urology, The Second Hospital of Tianjin Medical University, Tianjin Medical University, 23 Pingjiang Road, Tianjin 300211, P.R. China

E-mail: xuyong_2011@aliyun.com

Key words: microRNA-30a-5p, human renal cancer, phosphatase and tensin homolog, protein kinase $\mathrm{B}$, metadherin

\section{Introduction}

Renal cell carcinoma (RCC) is the most common space-occupying lesion in adult kidneys and accounts for $~ 3 \%$ of all tumors. In addition, the mortality rate of RCC is $\leq 40 \%$ (1). RCC is derived from renal proximal tubule cells, and clear cell carcinoma is the most common subtype, which accounts for $80-85 \%$ of metastatic RCC (2). With regards to treatment, RCC is not sensitive to radiotherapy or chemotherapy. Although surgical tumor resection is the optimal treatment strategy at present, it is associated with $20-40 \%$ postoperative relapse. Since RCC is resistant to radiotherapy and chemotherapy, effective postoperative adjuvant treatment for RCC is lacking (3). In addition, the lack of biochemical markers for the early diagnosis of RCC, as well as follow-up data, hinders the timely diagnosis of RCC. Therefore, there is an urgent need to understand the development of RCC at molecular and genetic levels, and to identify biochemical markers that can postoperatively predict the early metastasis of RCC (4).

MicroRNA(miRNA) expression profile screening is one of the most advanced methods used to research tumor-associated molecules (5). miRNAs are endogenous, non-coding, single-stranded RNAs. The mechanism of action of miRNAs is one kind of the epigenetic regulation and control mechanism, and miRNAs serve important roles in the regulation of gene expression (6). It has been estimated that one miRNA can regulate the expression of 100 target genes, and mRNAs of $>10,000$ genes are directly regulated by miRNAs (7). miRNAs are highly conserved among various species and regulate numerous biological functions (6). In addition, miRNAs are able to simultaneously regulate the expression of oncogenes and tumor suppressor genes through regulating critical cellular activities, including metabolism, differentiation, development and apoptosis. It has previously been reported that miRNAs serve a crucial regulatory role in carcinogenesis of prostate cancer, lung cancer, breast cancer and colon cancer, and miRNAs are also considered to be involved in the pathogenesis of RCC $(8,9)$. Numerous RCC-specific miRNAs have been identified through the screening of miRNA expression profiles, the majority of which exert their functions by disrupting the balance between oncogene and tumor suppressor 
gene expression (8). A recent study indicated that the regulatory mechanisms of miRNAs in RCC are complex, and are associated with numerous biological mechanisms, including mutations, epigenetic alterations, chromosomal abnormalities and deletions (10).

miRNA-30a-5p has been reported to inhibit metadherin (MTDH) expression, which serves an important role in tumor development. The MTDH gene is located in chromosome 8q22, and exhibits high expression in numerous tumor types, including breast cancer, prostate cancer, neuroblastoma and astrocytoma, where it is associated with poor prognosis (11). MTDH mediates several forms of chemotherapy resistance, and it has previously been demonstrated that the activation of MTDH expression can increase the death of tumor cells mediated by drugs, including cisplatin and doxorubicin (7). In addition, MTDH can enhance the invasion of tumor cells and increase the expression levels of relevant adhesion molecules via the nuclear factor- $\kappa \mathrm{B}$ pathway (12). MTDH is the downstream gene of Ha-ras, which induces upregulation of MTDH via the phosphoinositide 3-kinase (P13K)/protein kinase B (AKT)/glycogen synthase kinase 3//c-Myc pathway (13).

Among the numerous signal transduction pathways that mediate tumor cell apoptosis, the PI3K/phosphatase and tensin homolog (PTEN)/AKT signal transduction pathway is essential for growth regulation, and has an important role in the regulation of apoptosis. Activation of the PI3K/AKT signal transduction pathway can inhibit apoptosis induced by numerous stimuli and promote cell cycle progression, thus promoting cell survival and proliferation. Furthermore, this pathway participates in angiogenesis, serves an important role in tumor formation, and participates in tumor invasion and metastasis (14). As well as being able to induce the survival and differentiation of tumor cells, angiogenesis, and malignant development, the PI3K/AKT signal transduction pathway is associated with therapeutic resistance (15). At present, tumor treatments that target this pathway have gained extensive attention and abnormal activation of the PI3K/PTEN/AKT signaling pathway has been detected in several human tumor types, including lung cancer, pancreatic cancer, leukemia, liver cancer, multiple myeloma, prostate cancer and RCC (16). The present study aimed to investigate the effects of miRNA-30a-5p on tumor proliferation and to seek a potential therapeutic target for the treatment of human renal cancer.

\section{Materials and methods}

Tissue samples. A total of 229 patients with RCC from the Second Hospital of Tianjin Medical University (Tianjin, China) were included in the present study from March 2004 to October 2005; detailed clinicopathological characteristics of the patients are summarized in Table I. Renal parenchyma (RP) samples $(5 \mathrm{~cm})$ and RCC samples were collected from the patients. The RCC samples were divided into the following categories: TNM stage I + II, TNM stage III + IV and metastatic. The present study was approved by the Ethics Committee of The Second Hospital of Tianjin Medical University, Tianjin Medical University (Tianjin, China) and informed consent was obtained from all participants.

Reverse transcription-quantitative polymerase chain reaction (RT-qPCR). Total RNA was extracted from the cancer samples
Table I. Clinical characteristics of the patients.

\begin{tabular}{lc}
\hline Characteristic & $\mathrm{RCC}(\mathrm{n}=219)$ \\
\hline Gender & \\
Male & 122 \\
Female & 97 \\
Age at surgery [mean (range)] & $64(33-79)$ \\
TNM stage, n (\%) & \\
I & $76(34.70)$ \\
II & $27(12.33)$ \\
III & $99(45.20)$ \\
IV & $8(3.65)$ \\
Not available & $9(4.11)$ \\
Metastasis, n $(\%)$ & \\
M0 & $191(87.21)$ \\
M1 & $3(1.37)$ \\
NA & $25(11.42)$
\end{tabular}

and RP using TRIzol reagent (Invitrogen; Thermo Fisher Scientific, Inc., Waltham, MA, USA). Total RNA (500 ng) was reverse transcribed into cDNA using the PrimeScript RT reagent kit (Takara Bio, Inc., Otsu, Japan) according to the manufacturer's protocol. The miRNA-30a-5p, si-MTDH and negative plasmids were obtained from Tianjin Sainie Biologineering Technology Co., Ltd. (Tianjin, China). The sequence of miRNA-30a-5p was: miR-30a-5p, UGUAAACAU CCUCGACUGGAAG. qPCR was performed on an Applied Biosystems PCR7900 system (Applied Biosystems; Thermo Fisher Scientific, Inc.) using SYBR-Green PCR Master Mix reagent kit (Takara Bio, Inc.). U6 was used as an internal control. The qPCR thermocycling conditions were as follows: $95^{\circ} \mathrm{C}$ for $5 \mathrm{~min}$, followed by 40 cycles at $95^{\circ} \mathrm{C}$ for $15 \mathrm{sec}$, $57^{\circ} \mathrm{C}$ for $60 \mathrm{sec}$ and $72^{\circ} \mathrm{C}$ for $30 \mathrm{sec}$. Relative miRNA-30a-5p expression levels were calculated using the $2^{-\Delta \Delta \mathrm{Cq}}$ method (17).

Cell culture and transfection. Caki-2 cells, purchased from Shanghai Cell Bank of Chinese Academy of Sciences (Shanghai, China), were cultured in Dulbecco's modified Eagle's medium (DMEM) containing 10\% fetal bovine serum and $1 \%$ penicillin/streptomycin (all Gibco; Thermo Fisher Scientific, Inc.) at $37^{\circ} \mathrm{C}$ in a humidified atmosphere containing $5 \% \mathrm{CO}_{2}$ and $95 \%$ air. The miRNA-30a-5p and negative plasmids were obtained from Tianjin Sainie Biologineering Technology Co., Ltd. Caki-2 cells $\left(1 \times 10^{6}\right)$ were seeded in a 6-well plate and were transfected with $100 \mathrm{ng}$ miRNA-30a-5p and $100 \mathrm{ng}$ negative plasmids using Lipofectamine 2000 (Invitrogen; Thermo Fisher Scientific, Inc.). Transfected cells were maintained at $37^{\circ} \mathrm{C}$ in a $5 \% \mathrm{CO}_{2}$ atmosphere for $4 \mathrm{~h}$, and then old DMEM was removed and new DMEM was added into the cells at $37^{\circ} \mathrm{C}$ in a $5 \% \mathrm{CO}_{2}$ atmosphere.

MTT assay. Post-transfection for $24 \mathrm{~h}$, Caki-2 cells were adjusted to $1 \times 10^{4}$ cells $/ \mathrm{ml}$, inoculated in a 96 -well plate and cultured for an additional $48 \mathrm{~h}$. Subsequently, $10 \mu \mathrm{l}$ MTT was added to each well for $4 \mathrm{~h}$, after which dimethyl sulfoxide 
was added to each well for $20 \mathrm{~min}$. The absorbance of each well was detected at $490 \mathrm{~nm}$ using an Orion II microplate luminometer (Berthold Technologies GmbH \& Co. KG, Bad Wildbad, Germany). The overexpression of miR-30a-5p signifcantly suppressed cell proliferation and induced apoptosis of Caki- 2 cells at $48 \mathrm{~h}$.

Apoptosis assay. Post-transfection for 48 h, Caki-2 cells were adjusted to $1 \times 10^{6}$ cells $/ \mathrm{ml}$ and washed with PBS. Subsequently, the cells were incubated with $5 \mu$ l Annexin and $5 \mu$ l propidium iodide (both from BD Pharmingen, San Diego, CA, USA) for $15 \mathrm{~min}$ in the dark at room temperature. Flow cytometric analysis was conducted using a CyAn flow cytometer (Beckman Coulter, Miami, FL, USA) and the results were analyzed using ModFit (Verity Software House, Inc., Topsham, ME, USA).

Caspase-3 and caspase- 9 activity assay. Post-transfection for $48 \mathrm{~h}$, Caki-2 cells were adjusted to $1 \times 10^{6}$ cells $/ \mathrm{ml}$, inoculated in a 6-well plate and proteins were extracted using radioimmunoprecipitation assay (RIPA) buffer (Beyotime Institute of Biotechnology, Nanjing, China). Protein concentrations were determined using Micro Bicinchoninic Acid (BCA) Protein Assay kit (EMD Millipore, Billerica, MA, USA). Equal amounts of protein $(50 \mu \mathrm{g})$ were incubated with reagents from the caspase-3 (C1116) and caspase-9 (C1158) activities kit (Beyotime Institute of Biotechnology, Haimen, China) for $2 \mathrm{~h}$ at $37^{\circ} \mathrm{C}$. The absorbance of each well was detected at $405 \mathrm{~nm}$ using an Orion II microplate luminometer (Berthold Technologies $\mathrm{GmbH} \& \mathrm{Co} . \mathrm{KG})$.

Western blot analysis. Post-transfection for $48 \mathrm{~h}$, Caki-2 cells were adjusted to $1 \times 10^{6}$ cells $/ \mathrm{ml}$, inoculated in a 6 -well plate and proteins were extracted using RIPA buffer. Protein concentrations were determined using Micro BCA Protein Assay kit (EMD Millipore). Equal amounts of protein $(50 \mu \mathrm{g})$ were separated by $10 \%$ SDS-PAGE and transferred onto polyvinylidene fluoride membranes (EMD Millipore). After incubation with $5 \%$ non-fat milk in TBST for $1 \mathrm{~h}$ at $37^{\circ} \mathrm{C}$, the membranes were incubated with the following specific primary antibodies: B-cell lymphoma 2 (Bcl-2)-associated X protein (Bax; 1:2,000; 14796; Cell Signaling Technology, Inc., Beverly, MA, USA), Bcl-2 (1:1,000; 3498; Cell Signaling Technology, Inc.), MTDH (1:1,000; sc-517220; Santa Cruz Biotechnology, Santa Cruz, CA, USA), PTEN (1:1,000; sc-6817-R; Santa Cruz Biotechnology), phosphorylated (p)-AKT (1:1,000; sc-7985-R; Santa Cruz Biotechnology) and GAPDH (1:2,000; 5174; Cell Signaling Technology, Inc.) at $4^{\circ} \mathrm{C}$ overnight. Subsequently, membranes were incubated with an anti-rabbit horseradish peroxidase-conjugated secondary antibody $(1: 5,000 ; 7074$; Cell Signaling Technology, Inc.) for $1 \mathrm{~h}$ at $37^{\circ} \mathrm{C}$. An enhanced chemiluminescence western blotting detection system (EMD Millipore) was used to analyze protein expression levels. An enhanced chemiluminescence western blotting detection system (EMD Millipore) was used to analyze protein expression levels and results were semi-quantified using Image-ProPlus 6.0 software (Media Cybernetics, Inc., Rockville, MD, USA).

Immunocytofluorescence. Post-transfection for $12 \mathrm{~h}$, Caki-2 cells $\left(1 \times 10^{4}\right.$ cells $\left./ \mathrm{ml}\right)$ were seeded into cell chamber slides (Corning Life Sciences, Tewksbury, MA, USA) and were fixed with $4 \%$ formaldehyde. Subsequently, cells were incubated with anti-MTDH overnight at $4{ }^{\circ} \mathrm{C}$ for $24 \mathrm{~h}$ at room temperature, followed by incubation with the appropriate fluorescent secondary antibodies (1:500 dilution) at room temperature for $1 \mathrm{~h}$. Cells were also stained with DAPI at room temperature for $0.5 \mathrm{~h}$. Images were obtained using an FV1000 confocal microscope (Olympus Corporation, Center Valley, PA, USA).

Statistical analysis. Data are presented as the mean \pm standard deviation using SPSS $17.0(n=3)$. For analyzing significance between the various groups, one-way analysis of variance by Tukey's post test (for comparison among 3 groups) or Student's t-test (for comparison between 2 groups) was conducted. Overall survival (OS) and disease free survival (DFS) were analyzed using Kaplan-Meier test. $\mathrm{P}<0.05$ was considered to indicate a statistically significant difference.

\section{Results}

miRNA-30a-5p expression. The present study detected the expression levels of miRNA-30a-5p using RT-qPCR. As presented in Fig. 1A, miRNA-30a-5p expression was lower in RCC tissue samples compared with in RP tissue samples. In addition, miRNA-30a-5p expression was much lower in samples from patients with TNM stage III + IV RCC compared with in the RP tissue samples (Fig. 1B).

Overall survival (OS) and disease-free survival (DFS) are associated with miRNA-30a-5p expression. The present study also determined whether OS and DFS were associated with miRNA-30a-5p expression in patients with RCC. OS and DFS were increased in patients with RCC and high miRNA-30a-5p expression compared with in those with low miRNA-30a-5p expression. These results indicated that miRNA-30a-5p expression may affect RCC (Fig. 2).

Overexpression of miRNA-30a-5p suppresses cell proliferation and induces apoptosis of Caki-2 cells. To determine the effects of elevated miRNA-30a-5p expression on cell proliferation and apoptosis of Caki-2 cells, an MTT assay and flow cytometry were conducted. As presented in Fig. 3, overexpression of miRNA-30a-5p significantly suppressed cell proliferation and induced apoptosis of Caki-2 cells.

Overexpression of miRNA-30a-5p promotes caspase-3/9 activities in Caki-2 cells. To identify the potential genes that mediate the effects of miRNA-30a-5p on apoptosis of Caki-2 cells, caspase-3/9 activities were detected in Caki- 2 cells. Caspase-3/9 activities were significantly increased in Caki-2 cells transfected with miRNA-30a-5p compared with in the control group (Fig. 4).

Effects of miRNA-30a-5p overexpression on Bax and MTDH protein expression levels in Caki-2 cells. To determine whether miRNA-30a-5p expression had an effect on MTDH and Bax protein expression in Caki-2 cells, Bax and MTDH protein expression levels were analyzed using western blot analysis. The results indicated that overexpression of miRNA-30a-5p increased the protein expression levels of Bax and inhibited MTDH protein expression in Caki-2 cells (Fig. 5). 

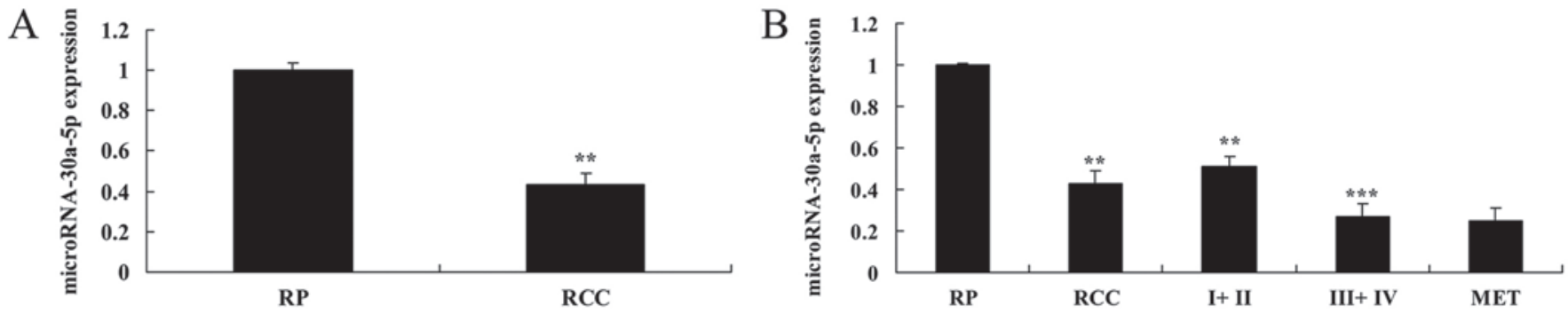

Figure 1. MicroRNA-30a-5p expression. (A) MicroRNA-30a-5p expression was detected in RP and RCC samples using RT-qPCR. (B) MicroRNA-30a-5p expression was detected in RP and RCC samples, which were divided into TNM stage I + II, TNM stage III + IV and MET samples, using RT-qPCR. ${ }^{* *} \mathrm{P}<0.05$ compared with RP, ${ }^{* * *} \mathrm{P}<0.05$ compared with RCC. MET, metastasis; RCC, renal cell carcinoma; RP, renal parenchyma; RT-qPCR, reverse transcription-quantitative polymerase chain reaction.
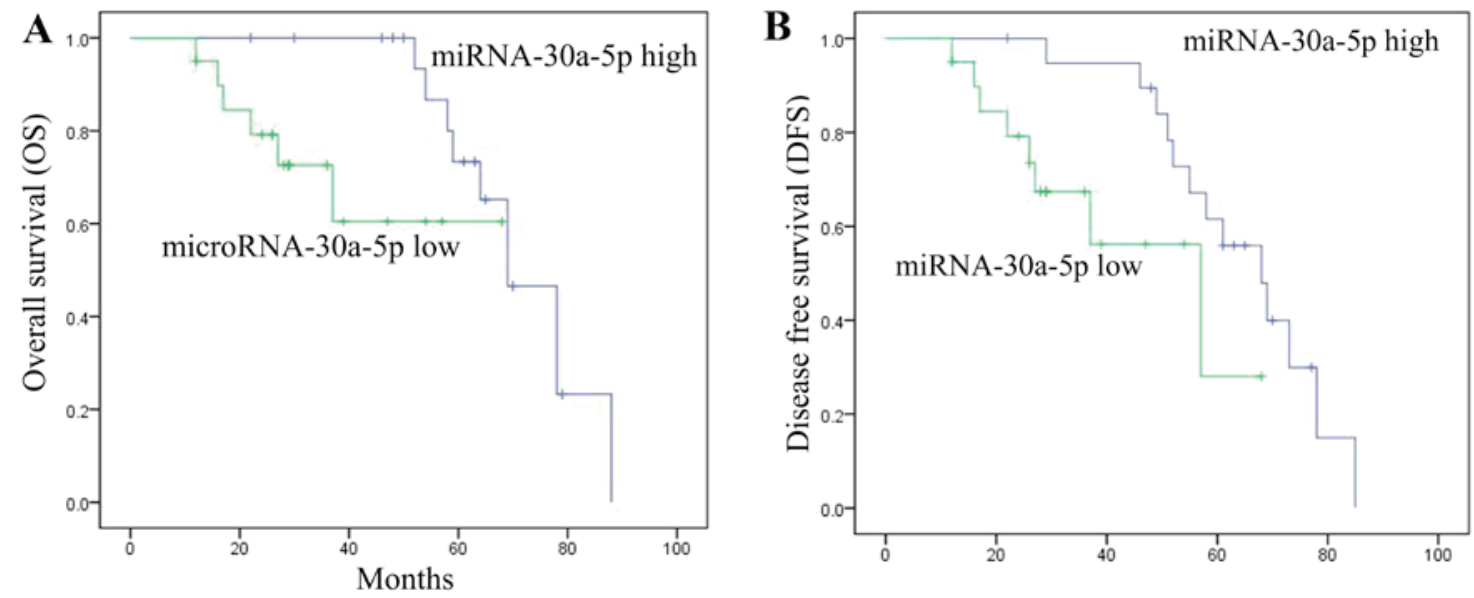

Figure 2. OS and DFS are associated with miRNA-30a-5p expression. (A) OS and (B) DFS were increased in patients with renal cell carcinoma and high miRNA-30a-5p expression. DFS, disease-free survival; miRNA-30a-5p, microRNA-30a-5p; OS, overall survival.
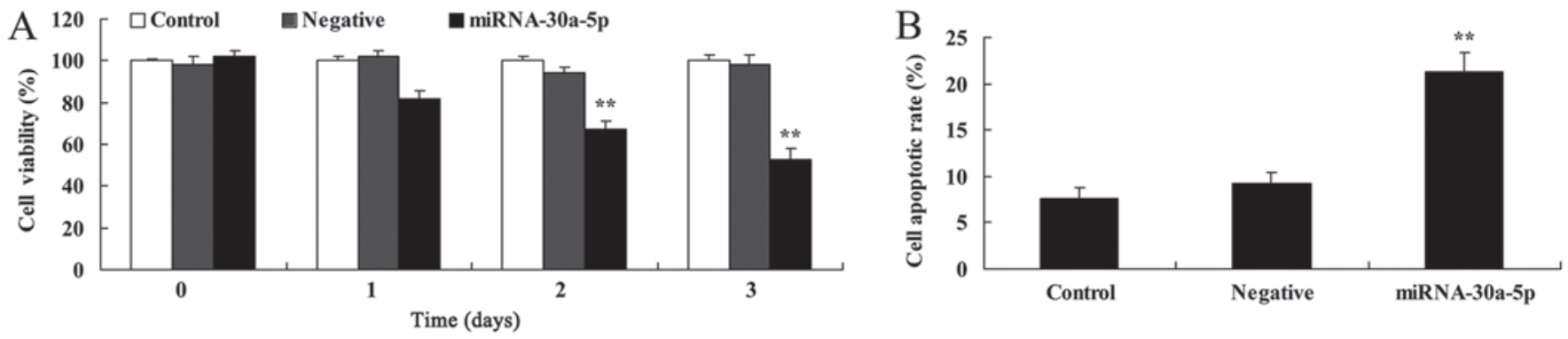

Figure 3. Overexpression of miRNA-30a-5p suppresses cell proliferation and induces apoptosis of Caki-2 cells. Overexpression of miRNA-30a-5p (A) suppressed cell proliferation and (B) induced apoptosis of Caki-2 cells, as determined by MTT assay and flow cytometry, respectively. Control, control group; Negative, negative control group; miRNA-30a-5p, miRNA-30a-5p overexpression group. ${ }^{* *} \mathrm{P}<0.05$ compared with the Control group. miRNA-30a-5p, microRNA-30a-5p.

Overexpression of miRNA-30a-5p inhibits MTDH protein expression in Caki-2 cells. The present study also analyzed whether miRNA-30a-5p affected MTDH protein expression in Caki-2 cells by immunocytofluorescence. Overexpression of miRNA-30a-5p markedly inhibited MTDH protein expression in Caki-2 cells, as determined using immunocytofluorescence (Fig. 6).

Overexpression of miRNA-30a-5p affects PTEN and $p-A K T$ protein expression in Caki-2 cells. In order to elucidate whether miRNA-30a-5p affects p-AKT and PTEN protein expression in Caki-2 cells, western blotting was conducted. As shown in Fig. 7, overexpression of miRNA-30a-5p significantly induced

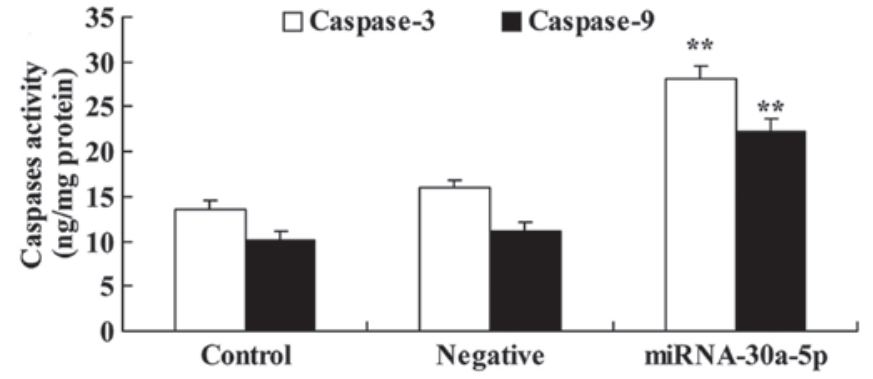

Figure 4. Overexpression of miRNA-30a-5p promotes caspase-3/9 activities in Caki-2 cells. Control, control group; Negative, negative control group; miRNA-30a-5p, miRNA-30a-5p overexpression group. ${ }^{* *} \mathrm{P}<0.05$ compared with the Control group. miRNA-30a-5p, microRNA-30a-5p. 
A

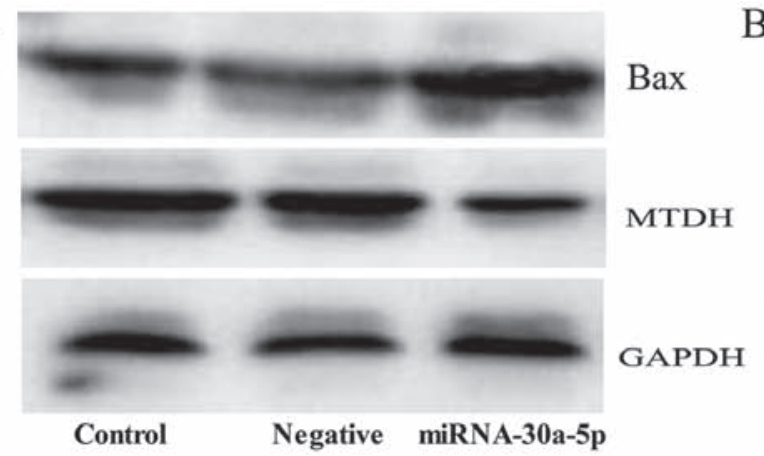

$\mathrm{C}$

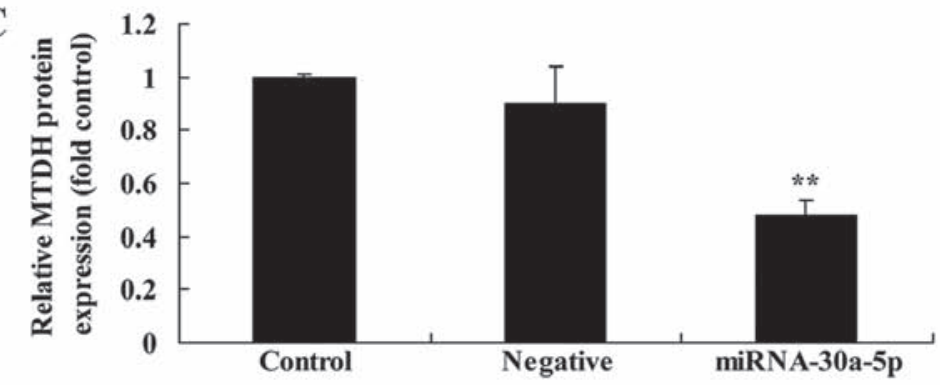

Figure 5. Effects of miRNA-30a-5p overexpression on Bax and MTDH protein expression levels in Caki-2 cells. (A) Bax and MTDH protein expression were determined by western blotting. Overexpression of miRNA-30a-5p (B) increased Bax and (C) inhibited MTDH protein expression levels in Caki-2 cells, as determined by statistical analysis. Control, control group; Negative, negative control group; miRNA-30a-5p, miRNA-30a-5p overexpression group. ${ }^{* *} \mathrm{P}<0.05$ compared with the Control group. Bax, B-cell lymphoma 2-associated X protein; miRNA-30a-5p, microRNA-30a-5p; MTDH, metadherin.

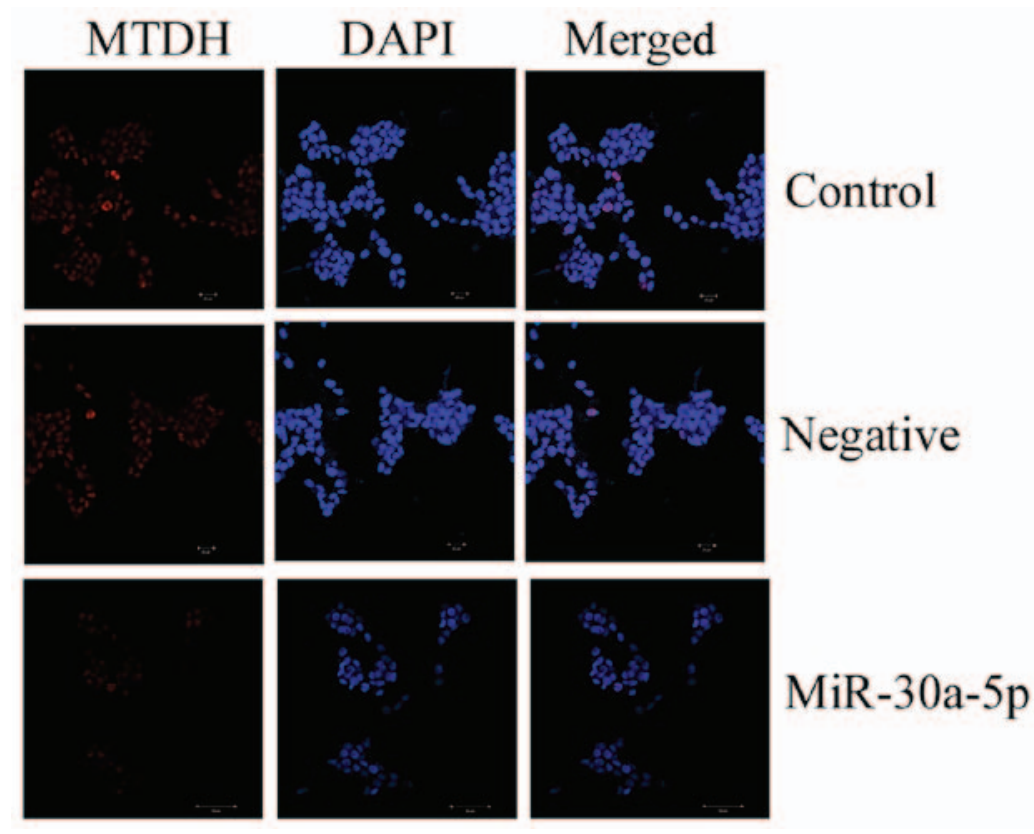

Figure 6. Overexpression of miRNA-30a-5p inhibits MTDH protein expression in Caki-2 cells, as determined using immunocytofluorescence (magnification, x5). Control, control group; Negative, negative control group; miRNA-30a-5p, miRNA-30a-5p overexpression group. miRNA/miR-30a-5p, microRNA-30a-5p; MTDH, metadherin.

the protein expression levels of PTEN and suppressed p-AKT protein expression in Caki-2 cells.

Small interfering RNA (si)-MTDH increases the effects of miRNA-30a-5p on MTDH protein expression in Caki-2 cells. The present study aimed to determine how MTDH regulates the effects of miRNA-30a-5p in RCC. As presented in Fig. 8, knockdown of MTDH, alongside miRNA-30a-5p overex- pression, significantly inhibited MTDH protein expression in Caki-2 cells compared with in the control group.

si-MTDH increases the effects of miRNA-30a-5p on inhibition of cell proliferation and promotion of apoptosis of Caki-2 cells. The present study aimed to determine whether si-MTDH affects miRNA-30a-5p-induced inhibition of cell proliferation and promotion of apoptosis of Caki-2 cells. As presented 
A

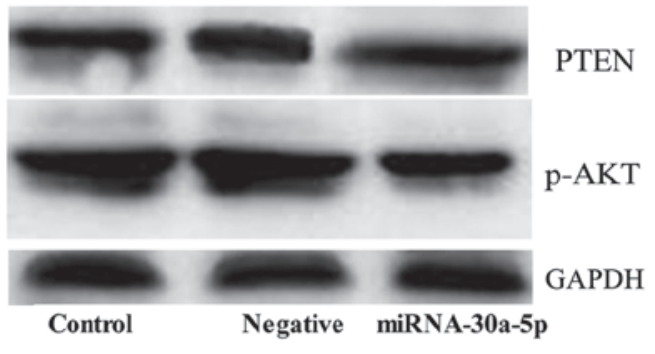

B

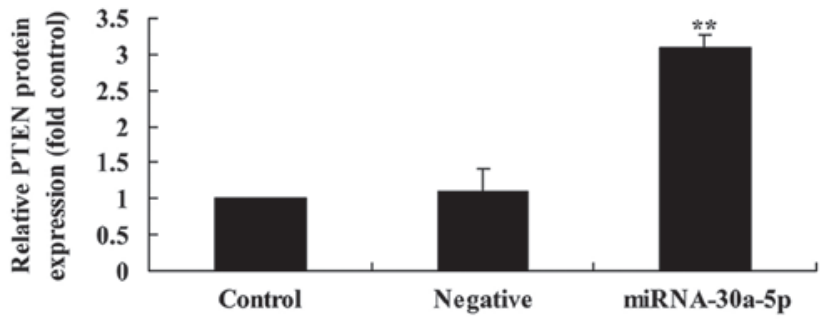

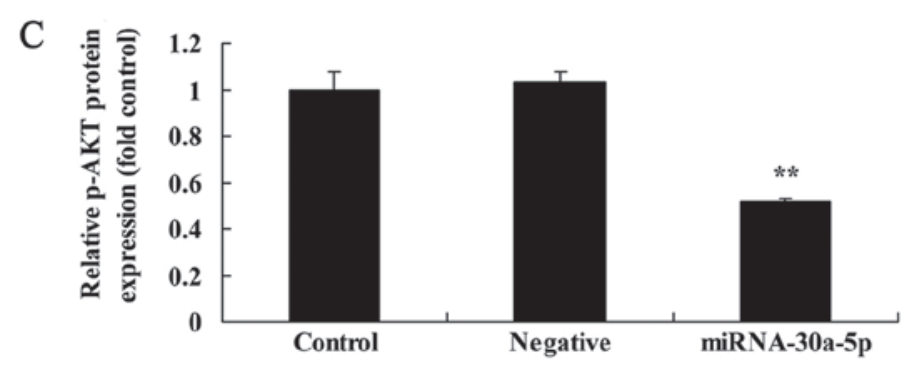

Figure 7. Overexpression of miRNA-30a-5p suppresses p-AKT protein expression levels in Caki-2 cells. (A) PTEN and p-AKT protein expression levels were determined by western blotting. Overexpression of miRNA-30a-5p (B) increased PTEN and (C) inhibited p-AKT protein expression levels in Caki-2 cells, as determined by statistical analysis. Control, control group; Negative, negative control group; miRNA-30a-5p, miRNA-30a-5p overexpression group. ${ }^{* *} \mathrm{P}<0.05$ compared with the Control group. miRNA-30a-5p, microRNA-30a-5p; p-AKT, phosphorylated-protein kinase B; PTEN, phosphatase and tensin homolog.

A

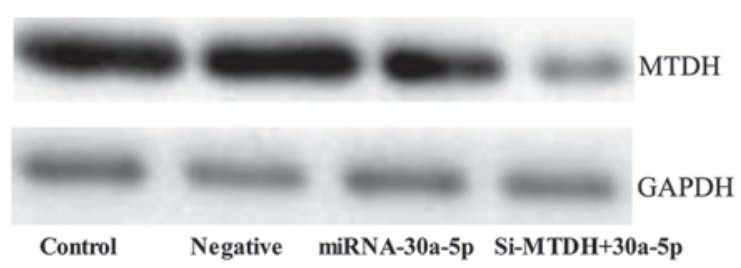

B

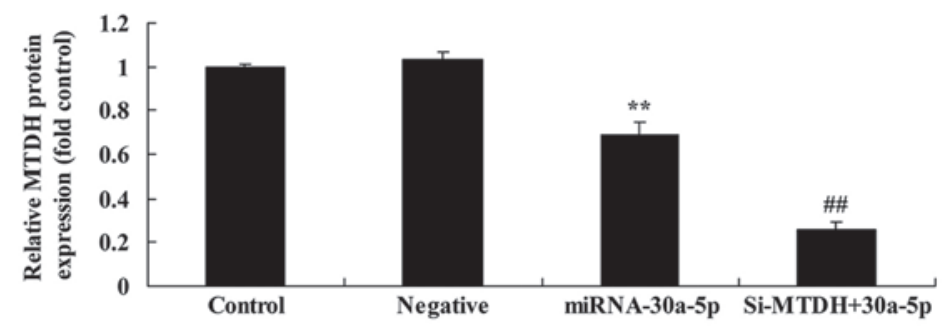

Figure 8. Knockdown of MTDH increases the effects of miRNA-30a-5p on MTDH protein expression in Caki-2 cells. si-MTDH increased the effects of miRNA-30a-5p on MTDH protein expression in Caki-2 cells, as determined using (A) western blotting and (B) statistical analysis. Control, control group; Negative, negative control group; miRNA-30a-5p, microRNA-30a-5p overexpression group; si-MTDH + 30a-5p, si-MTDH + miRNA-30a-5p overexpression group. ${ }^{* *} \mathrm{P}<0.05$ compared with the Control group; ${ }^{\# /} \mathrm{P}<0.05$ compared with the miRNA-30a-5p group. miRNA-30a-5p, microRNA-30a-5p; MTDH, metadherin; si-MTDH, small interfering RNA-MTDH.
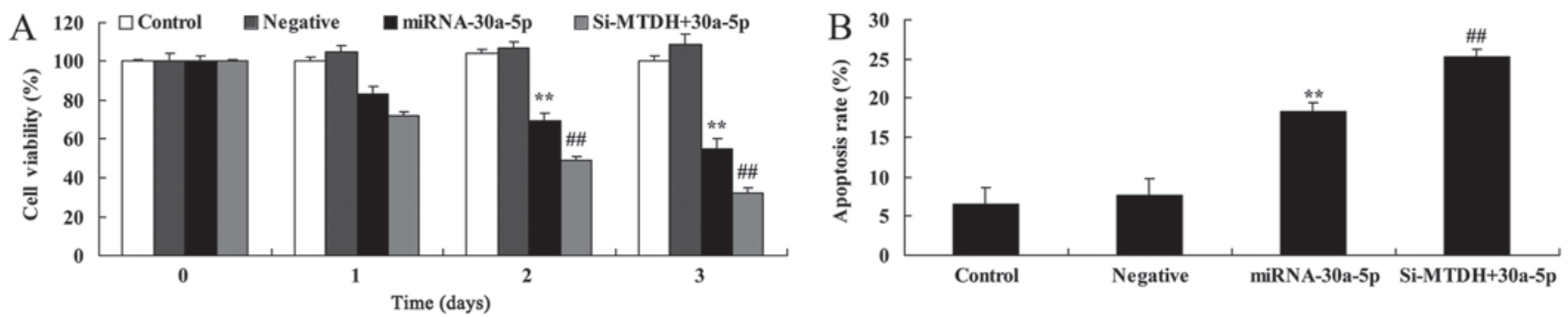

Figure 9. Knockdown of MTDH increases the effects of miRNA-30a-5p on the inhibition of cell proliferation and promotion of apoptosis of Caki-2 cells. si-MTDH increased the effects of miRNA-30a-5p on (A) inhibition of cell proliferation and (B) promotion of apoptosis of Caki-2 cells, as determined by MTT assay and flow cytometry, respectively. Control, control group; Negative, negative control group; miRNA-30a-5p, microRNA-30a-5p overexpression group; si-MTDH + 30a-5p, si-MTDH + miRNA-30a-5p overexpression group. ${ }^{* *} \mathrm{P}<0.05$ compared with the Control group; ${ }^{\# \# /} \mathrm{P}<0.05$ compared with the miRNA-30a-5p group. miRNA-30a-5p, microRNA-30a-5p; MTDH, metadherin; si-MTDH, small interfering RNA-MTDH.

in Fig. 9, knockdown of MTDH, alongside miRNA-30a-5p overexpression, inhibited cell proliferation and increased apoptosis of Caki-2 cells compared with in the control group.

si-MTDH increases the effects of miRNA-30a-5p on caspase-3/9 activities of Caki-2 cells. The present study investigated the mechanism underlying the effects of miRNA-30a-5p on Caki-2 cells; caspase-3/9 activities were analyzed using ELISA kits. Caspase-3/9 activities were significantly reduced in response to miRNA-30a-5p overexpression and knockdown of MTDH in Caki-2 cells compared with in the control group (Fig. 10). 


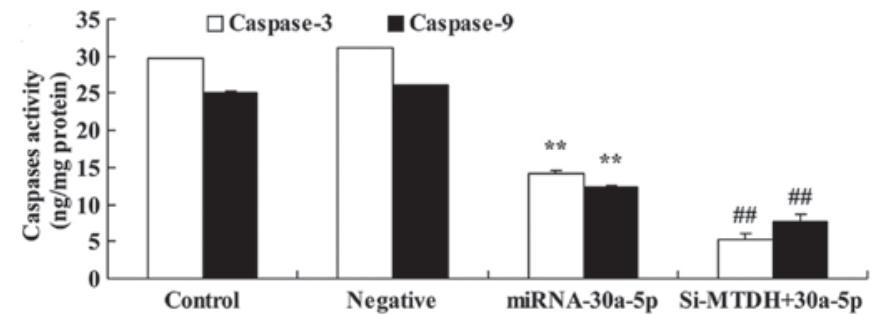

Figure 10. Knockdown of MTDH increases the effects of miRNA-30a-5p on caspase-3/9 activities in Caki-2 cells. Control, control group; Negative, negative control group; miRNA-30a-5p, microRNA-30a-5p overexpression group; si-MTDH + 30a-5p, si-MTDH + miRNA-30a-5p overexpression group. ${ }^{* *} \mathrm{P}<0.05$ compared with the Control group; ${ }^{\# \prime} \mathrm{P}<0.05$ compared with the miRNA-30a-5p group. miRNA-30a-5p, microRNA-30a-5p; MTDH, metadherin; si-MTDH, small interfering RNA-MTDH.

si-MTDH increases the effects of miRNA-30a-5p on Bax, PTEN and p-AKT protein expression in Caki-2 cells. The present study aimed to determined the effects of si-MTDH on miRNA-30a-5p-regulated expression of Bax, PTEN and p-AKT in Caki-2 cells. The protein expression levels of Bax and PTEN were significantly promoted, whereas p-AKT protein expression was significantly suppressed in Caki-2 cells in response to miRNA-30a-5p overexpression and MTDH knockdown compared with in the control group (Fig. 11).

\section{Discussion}

RCC is the most common type of kidney cancer, which accounts for $\sim 3 \%$ of all body tumors. In addition, the morbidity of RCC has increased in the past 20 years (18). Surgery remains the main treatment strategy for RCC; however, 30\% of patients develop recurrence within 3 years postsurgery. The 5 -year survival rate of RCC is $<10 \%$ and $\sim 25 \%$ of patients have metastasis at the time of diagnosis (19). Therefore, the identi- fication of reliable biological markers is of importance for the early diagnosis of RCC, the judgment of patient prognosis and the instruction of individualized treatment (20). miRNAs exert their important functions by influencing tumor proliferation, migration and invasion (8). The results of the present study demonstrated that miRNA-30a-5p expression was lower in tumor samples from patients with RCC compared with in RP tissue samples. In addition, miRNA-30a-5p expression was much lower in tumor samples from patients with TNM stage III + IV RCC compared with in RP tissue samples.

RCC is associated with numerous genes and its pathogenesis is complex; therefore, the molecular and biological foundation for its etiology and development remains unclear (21). However, with in-depth research regarding the molecular mechanisms underlying tumor cell growth, proliferation and apoptosis, it has been reported that an imbalance in the internal environment is an important factor for tumor development; the main cause of disturbances to the internal environment is imbalances in numerous intracellular signal pathways (22). It has previously been demonstrated that abnormalities in the signal transduction pathways that control cell proliferation and differentiation results in cellular growth disorders; in particular, increased signaling of pathways that promote cell proliferation, or reduced signaling of pathways that inhibit cell proliferation and promote cell apoptosis, at the cellular level during tumor formation may lead to tumor formation or reduced tumor cell apoptosis (23). In the present study, OS and DFS were increased in patients with RCC and high miRNA-30a-5p expression compared with in those with low miRNA-30a-5p expression.

The present study demonstrated that overexpression of miRNA-30a-5p inhibited MTDH, upregulated PTEN, and suppressed p-AKT protein expression levels in Caki-2 cells. Our results were similar to results from previous studies (24-26), which showed that miRNA-30a-5p regulated $\mathrm{MTDH} / \mathrm{PTEN} / \mathrm{AKT}$ signal pathway to induce renal cancer cell
A

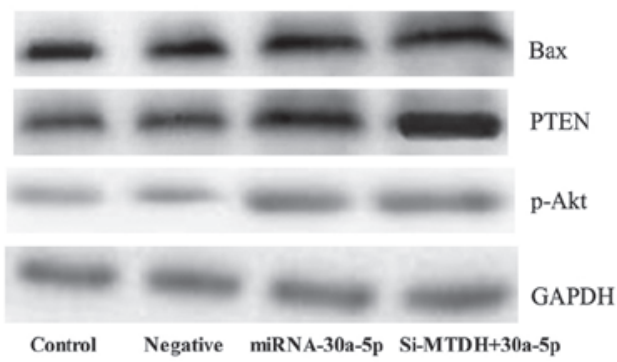

C

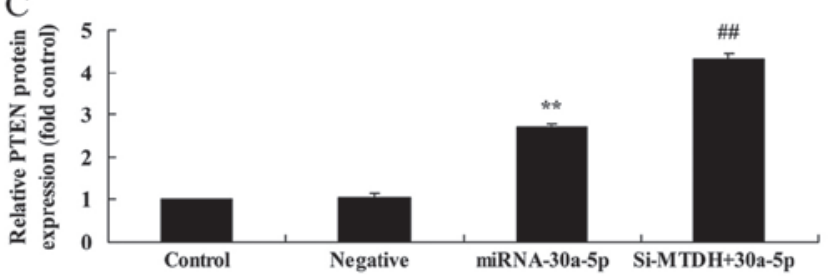

B

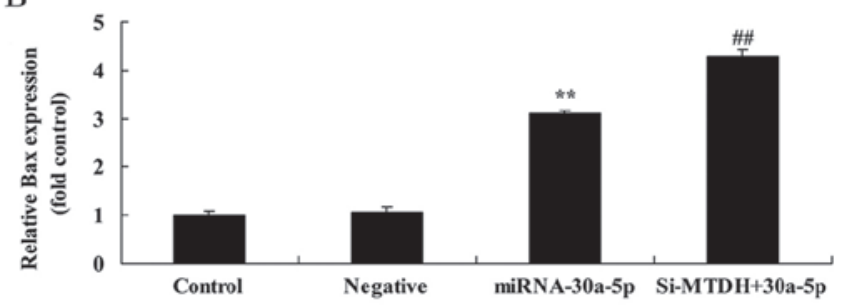

D

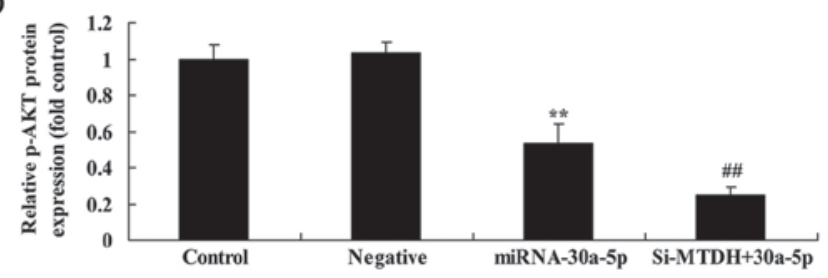

Figure 11. Knockdown of MTDH increases the effects of miRNA-30a-5p on Bax, PTEN and p-AKT protein expression in Caki-2 cells. si-MTDH increased the effects of miRNA-30a-5p on Bax, PTEN and p-AKT protein expression in Caki-2 cells, as determined using (A) western blot analysis and (B-D) statistical analysis. ${ }^{* *} \mathrm{P}<0.05$ compared with the Control group; ${ }^{\# /} \mathrm{P}<0.05$ compared with the miRNA-30a-5p group. Control, control group; Negative, negative control group; miRNA-30a-5p, microRNA-30a-5p overexpression group; si-MTDH + 30a-5p, si-MTDH + miRNA-30a-5p overexpression group. Bax, B-cell lymphoma 2-associated X protein; miRNA-30a-5p, microRNA-30a-5p; MTDH, metadherin; p-AKT, phosphorylated-protein kinase B; PTEN, phosphatase and tensin homolog; si-MTDH, small interfering RNA-MTDH. 
apoptosis. The present study de monstrated that overexpression of miRNA-30a-5p inhibited MTDH, upregulated PTEN, and suppressed p-AKT protein expression levels in Caki-2 cells. The present study demonstrated that overexpression of miRNA-30a-5p inhibited MTDH, upregulated PTEN, and suppressed p-AKT protein expression levels in Caki-2 cells, which showed that miRNA-30a-5p regulates MTDH/PTEN/ AKT pathway to suppress cell growth in human renal cancer.

A recent study demonstrated that the main function of AKT is the direct inhibition of cell apoptosis (27). The Bcl-2 family is also closely associated with cell apoptosis, numerous members of which, including Bcl-2-associated death promoter (Bad), exert proapoptotic effects. AKT can phosphorylate the Ser136/Ser112 residue of the Bad protein, thus resulting in its disaggregation from $\mathrm{Bcl}-2$ or Bcl-extra large, Bad then binds with the chaperonin 14-3-3, thus resulting in the upregulation of anti-apoptotic factors, including Bcl-2, A1, X-linked inhibitor of apoptosis protein and survivin, and the loss of its proapoptotic effect (28). The present study demonstrated that overexpression of miRNA-30a-5p may suppress cell proliferation, induce apoptosis, promote caspase-3/9 activities and increase Bax protein expression levels in Caki-2 cells.

Activated AKT can directly catalyze and phosphorylate Ser196 of caspase-9, resulting in its inactivation, thus reducing its proapoptotic effect. In addition, when the AKT kinase domain is activated, cells can resist penicillin-induced apoptosis, suggesting that only AKT with the complete kinase activity domain can promote the anti-apoptotic effect (29). The present study demonstrated that si-MTDH increased the effects of miRNA-30a-5p on the inhibition of cell proliferation and the promotion of apoptosis, caspase-3/9 activities and Bax protein expression in Caki-2 cells. The present study demonstrated that si-MTDH increased the effects of miRNA-30a-5p on the inhibition of cell proliferation and the promotion of apoptosis, caspase-3/9 activities and Bax protein expression in Caki-2 cells. MTDH is an important role in the anti-effect of miRNA-30a-5p on human renal cancer.

A previous study indicated that MTDH can activate numerous signaling pathways, regulate physiological and pathological cellular processes, and mediate the proliferation, invasion, metastasis, angiogenesis and chemotherapeutic resistance of tumor cells (30). MTDH is a downstream target gene of AKT, as well as an upstream activator of the PI3K/AKT pathway; PI3K/AKT and c-Myc can induce MTDH expression, which can further activate PI3K/AKT and upregulate c-Myc expression, resulting in the expression of N-Myc in a neuroblastoma cell line, further enhancing oncogenic effects, and forming a vicious cycle during tumor formation (31). In the present study suppression of MTDH expression upregulated PTEN and suppressed p-AKT protein expression levels in Caki-2 cells. In the present study suppression of MTDH expression upregulated PTEN and suppressed p-AKT protein expression levels in Caki-2 cells by miRNA-30a-5p. MTDH/PTEN/AKT pathway participated in the anti-effect of miRNA-30a-5p on human renal cancer.

In conclusion, the present study demonstrated that miRNA-30a-5p may suppress human RCC cell proliferation via the MTDH/PTEN/AKT pathway. Further studies aim to determine whether miRNAs can be practically applied in the treatment of RCC.

\section{Acknowledgements}

The present study was funded by the Application Base and FrontierTechnology Project to T.Z.(grantno.15JCQNJC10400), and was supported in part by the National Natural Science Foundation of China (grant no. 81301949) and the Natural Science Foundation of Tianjin (grant no. 16JCYBJC26500) to C.L., and the Natural Science Foundation of Hebei Province (grant no. H2015209164) to H.L.

\section{References}

1. Rini BI, Melichar B, Fishman MN, Oya M, Pithavala YK, Chen Y, Bair AH and Grünwald V: Axitinib dose titration: Analyses of exposure, blood pressure and clinical response from a randomized phase II study in metastatic renal cell carcinoma. Ann Oncol 26: 1372-1377, 2015.

2. Voss MH, Chen D, Marker M, Hakimi AA, Lee CH, Hsieh JJ, Knox JJ, Voi M and Motzer RJ: Circulating biomarkers and outcome from a randomised phase II trial of sunitinib vs everolimus for patients with metastatic renal cell carcinoma. $\mathrm{Br}$ J Cancer 114: 642-649, 2016.

3. Huijts CM, Santegoets SJ, van den Eertwegh AJ, Pijpers LS, Haanen JB, de Gruijl TD, Verheul HM and van der Vliet HJ: Phase I-II study of everolimus and low-dose oral cyclophosphamide in patients with metastatic renal cell cancer. BMC Cancer 11: 505, 2011.

4. Molina AM, Hutson TE, Larkin J, Gold AM, Wood K, Carter D, Motzer R and Michaelson MD: A phase 1b clinical trial of the multi-targeted tyrosine kinase inhibitor lenvatinib (E7080) in combination with everolimus for treatment of metastatic renal cell carcinoma (RCC). Cancer Chemother Pharmacol 73: 181-189, 2014.

5. Li Z, Chen Y, Hu S, Zhang J, Wu J, Ren W, Shao N and Ying X: Integrative analysis of protein-coding and non-coding RNAs identifies clinically relevant subtypes of clear cell renal cell carcinoma. Oncotarget 7: 82671-82685, 2016.

6. Zhang L, Xul B, Chen S, Lu K, Liu C, Wang Y, Zhao Y, Zhang X, Liu D and Chen M: The complex roles of microRNAs in the metastasis of renal cell carcinoma. J Nanosci Nanotechnol 13: 3195-3203, 2013

7. Wang P, Yin B, Shan L, Zhang H, Cui J, Zhang M and Song Y: RNA interference-mediated knockdown of astrocyte elevated gene-1 inhibits growth, induces apoptosis, and increases the chemosensitivity to 5 -fluorouracil in renal cancer Caki-1 cells. Mol Cells 37: 857-864, 2014.

8. Tutar Y: miRNA and cancer; computational and experimental approaches. Curr Pharm Biotechnol 15: 429, 2014.

9. Al-Ali BM, Ress AL, Gerger A and Pichler M: MicroRNAs in renal cell carcinoma: Implications for pathogenesis, diagnosis, prognosis and therapy. Anticancer Res 32: 3727-3732, 2012.

10. Mlcochova H, Machackova T, Rabien A, Radova L, Fabian P, Iliev R, Slaba K, Poprach A, Kilic E, Stanik M, et al: Epithelial-mesenchymaltransition-associated microRNA/mRNA signature is linked to metastasis and prognosis in clear-cell renal cell carcinoma. Sci Rep 6: 31852, 2016.

11. Luo Z, Hu X, Xiong H, Qiu H, Yuan X, Zhu F, Wang Y and Zou Y: A polysaccharide from Huaier induced apoptosis in MCF-7 breast cancer cells via downregulation of MTDH protein. Carbohydr Polym 151: 1027-1033, 2016.

12. Du C, Yi X, Liu W, Han T, Liu Z, Ding Z, Zheng Z, Piao Y, Yuan J, Han Y, et al: MTDH mediates trastuzumab resistance in HER 2 positive breast cancer by decreasing PTEN expression through an NFKB-dependent pathway. BMC Cancer 14: 869, 2014.

13. Lee SG, Su ZZ, Emdad L, Sarkar D, Franke TF and Fisher PB: Astrocyte elevated gene-1 activates cell survival pathways through PI3K-Akt signaling. Oncogene 27: 1114-1121, 2008.

14. Seo BR, Min KJ, Cho IJ, Kim SC and Kwon TK: Curcumin significantly enhances dual PI3K/Akt and mTOR inhibitor NVP-BEZ235-induced apoptosis in human renal carcinoma Caki cells through downregulation of p53-dependent Bcl-2 expression and inhibition of Mcl-1 protein stability. PLoS One 9: e95588, 2014.

15. Lim W, Jeong W and Song G: Delphinidin suppresses proliferation and migration of human ovarian clear cell carcinoma cells through blocking AKT and ERK1/2 MAPK signaling pathways. Mol Cell Endocrinol 422: 172-181, 2016. 
16. Ribback S, Cigliano A, Kroeger N, Pilo MG, Terracciano L, Burchardt M, Bannasch P, Calvisi DF and Dombrowski F: $\mathrm{PI} 3 \mathrm{~K} / \mathrm{AKT} / \mathrm{mTOR}$ pathway plays a major pathogenetic role in glycogen accumulation and tumor development in renal distal tubules of rats and men. Oncotarget 6: 13036-13048, 2015.

17. Livak KJ and Schmittgen TD: Analysis of relative gene expression data using real-time quantitative PCR and the 2(-Delta Delta C(T)) Method. Methods 25: 402-408, 2001.

18. Fournier LS, Oudard S, Thiam R, Trinquart L, Banu E, Medioni J Balvay D, Chatellier G, Frija G and Cuenod CA: Metastatic renal carcinoma: Evaluation of antiangiogenic therapy with dynamic contrast-enhanced CT. Radiology 256: 511-518, 2010.

19. Bracarda S, Porta C, Boni C, Santoro A, Mucciarini C, Pazzola A Cortesi E, Gasparro D, Labianca R, Di Costanzo F, et al: Could interferon still play a role in metastatic renal cell carcinoma? A randomized study of two schedules of sorafenib plus interferon-alpha 2a (RAPSODY). Eur Urol 63: 254-261, 2013.

20. Li HC, Li JP, Wang ZM, Fu DL, Li ZL, Zhang D, Gan WM and Chong T: Identification of angiogenesis-related miRNAs in a population of patients with renal clear cell carcinoma. Oncol Rep 32: 2061-2069, 2014.

21. Su Z, Ni L, Yu W, Yu Z, Chen D, Zhang E, Li Y, Wang Y, Li X, Yang S, et al: MicroRNA-451a is associated with cell proliferation, migration and apoptosis in renal cell carcinoma. Mol Med Rep 11: 2248-2254, 2015.

22. Zheng J, Qin W, Jiao D, Ren J, Wei M, Shi S, Xi W, Wang H, Yang AG, Huan Y, et al: Knockdown of COUP-TFII inhibits cell proliferation and induces apoptosis through upregulating BRCA1 in renal cell carcinoma cells. Int J Cancer 139: 1574-1585, 2016.

23. Liang T, Hu XY, Li YH, Tian BQ, Li ZW and Fu Q: MicroRNA-21 regulates the proliferation, differentiation, and apoptosis of human renal cell carcinoma cells by the mTOR-STAT3 signaling pathway. Oncol Res 24: 371-380, 2016.

24. Yamada T, Horinaka M, Shinnoh M, Yoshioka T, Miki T and Sakai T: A novel HDAC inhibitor OBP-801 and a PI3K inhibitor LY294002 synergistically induce apoptosis via the suppression of survivin and XIAP in renal cell carcinoma. Int J Oncol 43 1080-1086, 2013.
25. Zhou J, Zhu G, Huang J, Li L, Du Y, Gao Y, Wu D, Wang X, Hsieh JT, He D, et al: Non-canonical GLI1/2 activation by PI3K/AKT signaling in renal cell carcinoma: A novel potential therapeutic target. Cancer Lett 370: 313-323, 2016.

26. Han X, Yang J, Jia Z, Wei P, Zhang H, Lv W, Sun J and Huo Q: Knockdown of serine-arginine protein kinase 1 inhibits the growth and migration in 6 renal cell carcinoma cells. Oncol Res 25: 389-395, 2017.

27. Papadopoulos EI, Yousef GM and Scorilas A: Gemcitabine impacts differentially on bladder and kidney cancer cells: Distinct modulations in the expression patterns of apoptosis-related microRNAs and BCL2 family genes. Tumour Biol 36: 3197-3207, 2015.

28. Burke MT, Morais C, Oliver KA, Lambie DL, Gobe GC, Carroll RP, Staatz CE, Sinnya S, Soyer HP, Winterford C, et al: Expression of Bcl-xL and Mcl-1 in the nonmelanoma skin cancers of renal transplant recipients. Am J Clin Pathol 143: 514-526, 2015.

29. Zhong WF, Wang XH, Pan B, Li F, Kuang L and Su ZX: Eupatilin induces human renal cancer cell apoptosis via ROS-mediated MAPK and PI3K/AKT signaling pathways. Oncol Lett 12: 2894-2899, 2016.

30. Zhu GC, Yu CY, She L, Tan HL, Li G, Ren SL, Su ZW, Wei M, Huang DH, Tian YQ, et al: Metadherin regulation of vascular endothelial growth factor expression is dependent upon the PI3K/Akt pathway in squamous cell carcinoma of the head and neck. Medicine (Baltimore) 94: e502, 2015.

31. Li WF, Ou Q, Dai H and Liu CA: Lentiviral-mediated short hairpin RNA knockdown of MTDH inhibits cell growth and induces apoptosis by regulating the PTEN/AKT pathway in hepatocellular carcinoma. Int J Mol Sci 16: 19419-19432, 2015. 